\title{
Prédictions des éclosions de maladies trar
par les moustiques selon les prévisions météorologiques au Canada
}

\author{
NH Ogden ${ }^{1 *}$, LR Lindsay², A Ludwig ${ }^{1}$, AP Morse ${ }^{3}, \mathrm{H}$ Zheng $^{4}$, H Zhu ${ }^{5}$
}

\section{Résumé}

Les systèmes d'alerte précoce permettant de prédire les éclosions de maladies infectieuses ont été reconnus comme étant une réponse adaptative clé aux changements climatiques. On s'attend à ce que le réchauffement, la variabilité climatique et les phénomènes météorologiques extrêmes associés aux changements climatiques mènent à une augmentation de la fréquence et de l'intensité des maladies transmises par les moustiques dans le monde entier, ce qui se traduira au Canada par un risque accru d'éclosions de maladies transmises par les moustiques endémiques et émergentes comme le virus du Nil occidental et d'autres maladies transmises par les moustiques. La rapidité de la diffusion d'informations sur le risque imminent d'éclosions de maladies transmises par les moustiques a d'importantes implications sanitaires pour le public, en ce qu'elle permet la mise en œuvre de mesures de contrôle des moustiques et des communications ciblées concernant la nécessité de mesures personnelles de protection - avant l'éclosion de la maladie. Le Canada a mis au point des modèles mécanistes et statistiques fondés sur les données météorologiques afin de prédire les éclosions de virus du Nil occidental, y compris des modèles pour différentes espèces de moustiques vectrices du virus du Nil occidental dans différentes régions du Canada. Bien que les résultats initiaux soient prometteurs, leur mise en œuvre à grande échelle nécessite l'évaluation et la validation approfondies de leurs aptitudes prévisionnelles. Les prévisions fondées sur les données météorologiques pour d'autres maladies transmises par les moustiques émergentes au Canada, comme l'encéphalite équine de l'Est, peuvent également être mises à contribution.

Citation suggérée : Ogden NH, Lindsay LR, Ludwig A, Morse AP, Zheng H, Zhu H. Prédictions d'éclosions de maladies transmises par les moustiques fondées sur les pronostics météorologiques au Canada. Relevé des maladies transmissibles au Canada 2019;45(5):141-7. https://doi.org/10.14745/ccdr.v45i05a03f

Mots-clés : maladies transmises par les moustiques, prévisions fondées sur les données météorologiques, virus du Nil occidental, encéphalite équine de l'Est, changements climatiques, Canada

\section{Introduction}

Le Groupe d'experts intergouvernemental des Nations Unies sur l'évolution du climat a identifié une stratégie d'adaptation clé permettant de faire face aux risques des changements climatiques pour la santé, soit la mise au point de systèmes d'alerte précoce (1). Un type de système d'avertissement précoce est la prédiction du risque basée sur les prévisions métérologiques c'est-à-dire d'utiliser les données météorologiques pour prédire le risque d'une éclosion de maladies infectieuses particulières dans une région précise. La recherche sur une grande gamme de maladies transmises par les moustiques, incluant le virus du Nil occidental, la malaria et la fièvre de la vallée du Rift, ont permis de prouver l'efficacité du concept des prévisions basées sur les météorologiques (2). La National Oceanic and Atmospheric Administration des États-Unis a lancé un programme pour faciliter la validation de divers modèles prévisionnels (3).

Parmi les maladies transmises par les moustiques, la plus courante au Canada est celle du virus du Nil occidental qu'on retrouve à l'état endémique dans le sud du pays, et des cas humains ont été recensés presque partout au pays. Chaque année, le virus cause de nombre cas dont la sévérité varie de légère à grave et allant même parfois jusqu'au décès (4). D'autres maladies transmises

Cette oeuvre est mise à la disposition selon les termes de la licence internationale Creative Commons Attribution 4.0

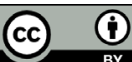

Affiliations

${ }^{1}$ Laboratoire national de microbiologie, Agence de la santé publique du Canada, Saint-Hyacinthe (Québec)

\section{${ }^{2}$ Laboratoire national de microbiologie, Agence de la santé publique du Canada, Winnipeg (Manitoba) \\ ${ }^{3}$ School of Environmental \\ Sciences, Université de Liverpool, Liverpool (Royaume-Uni) \\ ${ }^{4}$ Centre des maladies infectieuses d'origine alimentaire, environnementale et zoonotique, Agence de la santé publique du Canada, Ottawa (Ontario)}

${ }^{5}$ Department of Mathematics and Statistics and Laboratory of Mathematical Parallel Systems, York University, Toronto (Ontario)

\section{${ }^{\star}$ Correspondance :}

nicholas.ogden@canada.ca 
par les moustiques, y compris les virus du sérogroupe Californie (Jamestown Canyon et Snowshoe hare) et le virus de Cache Valley, sont endémiques au Canada. II existe des preuves que ces maladies sont plus fréquentes chez les humains au Canada que supposé $(5,6)$, et que leur prévalence pourrait augmenter à cause des changements climatiques (7). De plus, d'autres maladies transmises par les moustiques, endémiques à l'heure actuelle aux États-Unis, pourraient migrer vers le nord, soit au Canada, à la faveur des changements climatiques (8), ce qui suscite des inquiétudes croissantes. Il existe également un risque accru que d'autres maladies exotiques transmises par les moustiques, comme la dengue et la fièvre Chikungunya - et les moustiques qui les transmettent - $s$ 'établissent au Canada. En raison des changements climatiques, ces moustiques exotiques, qui ont été introduits suite à l'intensification des transports, des échanges de denrées et de matériel à l'échelle internationale, trouvent maintenant au Canada les conditions environnementales dont ils ont besoin pour survivre (9).

Un grand nombre de maladies transmises par les moustiques présentent un comportement épidémique de type sporadique à l'échelle de la planète (10). Ce profil épidémiologique est observé chez le virus du Nil occidental au Canada, ce qui signifie que des épidémies sont présentes certaines années, mais que la maladie se fait discrète d'autres années, où l'on n'observe qu'un petit nombre de cas. En 2007, 2215 cas ont été déclarés (la plupart recensés lors d'une éclosion dans les provinces des Prairies), en 2010, seulement cinq cas l'ont été et, en 2012, 428 cas (la plupart résultant d'une épidémie en Ontario et au Québec) (4). On croit que cette sporadicité découle surtout des effets de la température sur le cycle de vie du moustique et la transmission du virus du Nil occidental (voir ci-dessous). En 2007, un hiver doux suivi d'un printemps chaud et humide ont fourni les conditions idéales pour le foisonnement de l'espèce Culex tarsalis (le principal vecteur du virus du Nil occidental dans les Prairies), alors que la température torride dans l'est du Canada en 2012 peut être suspectée d'avoir accéléré et amplifié la transmission du virus par $C x$. pipiens et $C x$. restuans (les principaux vecteurs dans l'est du Canada) (4).

Les méthodes utilisées actuellement pour le contrôle du virus du Nil occidental au Canada consistent à surveiller le moustique (détection des niveaux de risque environnemental), à détecter l'infection chez les animaux sentinelles et à assurer la surveillance humaine (qui permet également d'évaluer la gravité du fardeau de la maladie). Le risque environnemental se mesure par le nombre de moustiques présents dans une zone donnée qui sont infectés et susceptibles de piquer les humains (4). Des hausses tant du nombre de moustiques infectés que du nombre de cas humains se produisent ordinairement de la fin de l'été au début de l'automne (4). Si ces hausses se produisent tôt en saison ou que les augmentations du nombre de moustiques infectés ou de cas humains sont plus importantes que d'habitude, ces chiffres peuvent indiquer qu'une épidémie est sur le point de se produire. Lorsqu'il existe un risque accru lié au virus du
Nil occidental, deux types d'interventions locales de gestion du risque pour la santé publique sont lancées. La première comprend l'utilisation de larvicides et (là où c'est acceptable) d'adulticides (11) afin de contrôle l'abondance des populations de moustiques, et la deuxième intervention vise à sensibiliser du public, en faisant la promotion de mesures de protection personnelle (12).

Le système de surveillance actuel a un désavantage, celui de ne pouvoir détecter les éclosions qu'une fois qu'elles se déclarent. En prenant en compte les effets du climat sur les cycles de vie du moustique et la transmission du virus, il est possible en théorie de prédire les éclosions de maladie causée par les moustiques et de déclencher une intervention des autorités de santé publique avant que l'éclosion ne se déclare $(3,13)$.

Cet article a pour but de décrire les concepts, les méthodes et le statut des prévisions de risque associés au virus du Nil occidental basées sur les prévisions météorologiques au Canada et de déterminer quelles sont les prochaines étapes requises pour la mise en œuvre de ces prévisions en tant qu'outil pour la santé publique.

\section{Concepts-clés des modèles prédictifs basés sur les prévisions météorologiques}

La prédiction du risque d'exposition au maladies transmises par les moustiques basée sur les prévisions météorologiques utilise les connaissances sur l'influence de la température ambiante et des précipitations sur le taux de survie et les cycles de vie des moustiques. Par exemple, étant donné que les températures ambiantes ont une influence sur la croissance des œufs, des larves et des pupes, les températures plus élevées accélèrent le cycle de vie du moustique ainsi que la période d'incubation extrinsèque, ou la vitesse à laquelle les pathogènes transmis par le moustique adulte se multiplient et passent de son système digestif aux glandes salivaires où ils sont transmis aux humains (14). Les températures élevées influencent également le niveau d'activité des moustiques adultes. En plus des changements de température, les fluctuations des précipitations peuvent également modifier la taille de la population de moustiques. Les précipitations excessives génèrent souvent des zones d'eau stagnante nécessaire au développement des larves et des pupes, ce qui peut faire augmenter le taux de reproduction des moustiques. A contrario, les sécheresses font en sorte que l'on trouve, en zones urbaines et périurbaines, de l'eau stagnante dans les canaux de drainage où le moustique peut se reproduire. En conséquence, certaines éclosions dans ces régions sont associées tant à la sécheresse qu'aux températures élevées. 
Les prédictions du risques basées sur les prévisions météorologiques examinent les conditions associées aux éclosions de maladies transmises par des moustiques, et ces conditions correspondent à un moustique particulier, à une maladie particulière transmise par un moustique dans une zone géographique particulière. La figure 1 illustre l'éclosion hypothétique d'une maladie transmise par les moustiques, avec ou sans intervention des autorités de santé publique grâce aux prédictions du risque basées sur les prévisions météorologiques. Selon les techniques de surveillance traditionnelle utilisées, il peut s'écouler d'une à quatre semaines entre le début d'une éclosion et sa confirmation (une semaine pour procéder à l'identification des moustiques infectés et quatre semaines pour le diagnostic clinique, les tests de laboratoire et la signalisation d'un patient humain infecté). (figure 1A). Ainsi, au moment où l'éclosion est confirmée, elle est en fait terminée.

Figure 1: Comment les prédictions du risque basées sur les prévisions métérologiques facilitent l'intervention précoce des autorités sanitaires publiques face à une éclosion de maladies transmises par les moustiques

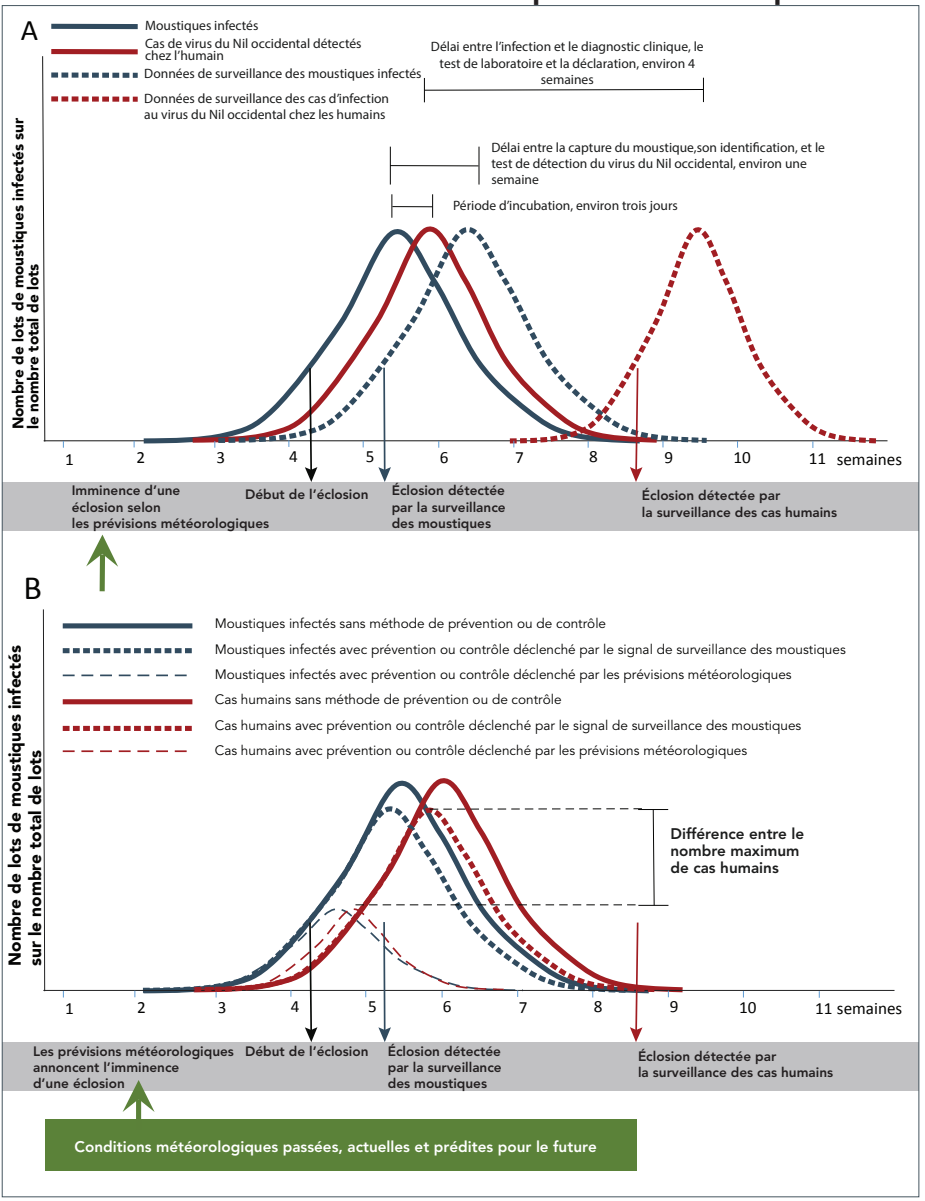

Légende de la figure : dans le diagramme A, la ligne continue indique le nombre de moustiques infectés et d'infections chez les humains en temps réel. La ligne pointillée indique le nombre de moustiques infectés et d'infections chez les humains au moment de leur détection par des systèmes de surveillance. Les lignes horizontales indiquent les raisons et la durée des délais entre les données en temps réel et leur détection au moyen de la surveillance. Dans le diagramme $B$, on compare le nombre de moustiques infectés et d'infections chez los humains en l'absence $d^{\prime}$ intervention en santé publique (ligne continue) au nombre d'interventions qui sont lancées à la suite des signaux émis grâce à la surveillance des moustiques (lignes pointillées) ou les prévision météorologique (lignes en tireté). La flèche noire indique le début d'une éclosion; les flèches de couleurs verte, bleue et rouge indiquent les signaux d'une éclosion qui sont respectivement fournis par les prévisions météorologiques et la surveillance des moustiques et des cas humains. Le rectangle vert indique les prévisions météorologiques actuelles et passées qui servent à mettre au point les données prévisionnelles du nombre de moustiques.
En comparaison, la figure 1B illustre les effets possibles de l'existence d'un signal de risque précoce basé sur les prévisions météorologiques, suivi de l'intervention précoce des autorités sanitaires publiques visant à réduire le nombre de moustiques infectés et la gravité de l'éclosion de maladies transmises par les moustiques (figure 1B).

\section{Types de modèles prédictifs}

Les prédictions du risque basées sur les prévisions métérologiques ont été établies à l'aide de modèles mécanistes ou statistiques. Les modèles prédictifs mis au point au Canada sont décrits ci-dessous.

\section{Modèles mécanistes}

Les modèles de type mécaniste les plus simples sont ceux qui utilisent des indices de température, obtenus à partir d'essais en laboratoire, pour désigner les étapes importantes du cycle de vie du moustique (p. ex. limites de l'activité du moustique) ou les cycles de transmission du virus. Une méthode (15), élaborée et mise en œuvre en Saskatchewan, se sert d'une simple mesure du nombre de degré-jours accumulés pendant lesquels la température est supérieure à $14,3^{\circ} \mathrm{C}$, soit la température à partir de laquelle le virus du Nil occidental se multiplie dans l'organisme de son vecteur dans les Prairies : le moustique à pattes rayées $(C x$. tarsalis). Ces données servent ensuite à prévoir la période pendant laquelle le moustique représente un risque élevé pour le public. Des calculs similaires sont utilisées dans des bulletins d'information publique diffusés par des organismes de santé publique au Québec et en Ontario, bien qu'elles n'aient pas été encore évaluées en tant que systèmes d'alerte précoce $(16,17)$. La plupart des modèles de type mécaniste sont des reconstructions mathématiques des cycles de vie du moustique et des cycles de transmission du pathogène. Ces modèles intègrent les effets connus de la température et des précipitations au cycle de vie du moustique, et les effets de la température sur la période d'incubation extrinsèque, de manière à prédire comment les conditions météorologiques récentes et prévues pourront influencer le nombre de moustiques et la proportion d'entre eux qui seront infectés au cours des prochaines semaines par le virus afin de prévoir le risque que courent les humains d'être exposés à un moustique infecté (18). Afin de s'assurer que les modèles prédisent de façon juste et efficace le risque dans un lieu en particulier à fin de mise en œuvre de mesure de prévention et de contrôle, ils devront comporter un grand nombre de paramètres détaillés responsables de l'estimation de facteurs dépendant des conditions météorologiques ou non qui auront une influence sur les cycles de vie du moustique et les cycles de transmission du pathogène. Historiquement, la mise au point de ces modèles visait surtout à prévoir les éclosions de maladies transmises par des moustiques exotiques, comme la malaria en Afrique (19). Au Canada, il n'existe à l'heure actuelle que des connaissances limitées sur l'influence de la température et de la pluie sur différents cycles de vie du moustique. 
Un seul modèle de prévisions météorologiques de type mécaniste intégrant la reconstruction des cycles de vie du moustique a été mis au point au Canada. Yu et al. ont mis au point un modèle de type mécaniste du cycle de vie des moustiques Cx. pipiens et Cx. restuans dans l'est du Canada (20), qui intègre les données sur les effets de la température sur la croissance et la survie du moustique. Ce modèle a bien réussi à refléter les données sur la surveillance du moustique qui ont été utilisées pour sa validation.

\section{Modèles statistiques}

Les modèles statistiques se servent du concept de I'« appariement de formes » pour déterminer quelle influence ont les températures et les pluies actuelles et récentes sur le nombre de moustiques et la proportion d'entre eux qui sont infectés de pathogènes $(21,22)$. L'appariement de formes se base sur les données météorologiques récentes et les données actuelles concernant la surveillance du moustique de manière à établir la relation quantitative entre le nombre de moustiques (et les proportions de moustiques qui sont infectés) un jour donné et les précipitations accumulées ou les températures et les précipitations moyennes pour des sites de capture particuliers au cours des semaines et des mois précédents. À l'aide de cette relation et compte tenu des données météorologiques prévues pour les semaines suivantes (données qui ont été tirées des modèles de prévisions météorologiques), on pourra prévoir le nombre de moustiques et la proportion d'entre eux qui sont infectés (23).

Au Canada, quatre modèles statistiques de prévisions météorologiques pour le virus du Nil occidental ont été mis au point, soit dans trois régions où le risque est le plus élevé : les régions méridionales des provinces des Prairies, le sud de I'Ontario et le sud du Québec. En Saskatchewan, on a établi un modèle prédisant tant le nombre de moustiques $C x$. tarsalis que la proportion de moustiques infectés dans les Prairies, les données sur la température et les précipitations servant de paramètres de prévision (23). Les prévisions du modèle ont été simplement comparées au nombre de cas humains une année donnée sur une période de deux mois à fin de validation; il a permis de prévoir le risque pendant ces deux mois selon une configuration spatiale, risque qui correspondait aux tendances observées pour l'incidence de cas humains. Les trois autres modèles statistiques ont été mis au point au Québec $(22)$ et en Ontario $(21,24)$ où les moustiques vecteurs du virus du Nil occidental sont $C x$. pipiens et $C x$. restuans. Tous les modèles ont utilisé les données sur la température et les précipitations comme variables explicatives, et on les a validés en les comparant aux données sur la surveillance des moustiques $(21,22,24)$. L'un des modèles de l'Ontario $(21)$ a été testé dans la région de Toronto par le centre de santé publique de la région de Peel (25). L'incidence des risques observés et prédits au cours de la période d'essai était faible.

\section{Approches en matière de validation}

Un certain nombre d'approches différentes existent pour valider des modèles de prévisions fondées sur les données météorologiques. Une de ces approches compare les données issues de méthodes de surveillance traditionnelle (de moustiques, de virus et de cas humains) avec les valeurs prédites par le modèle statistique qui ont été obtenues à l'aide de modèles mécanistes ou statistiques basés sur les prédictions météorologiques locales. Concernant les études menées au Canada à ce jour, cette validation entomologique laisse croire que les deux approches des prévisions fondées sur les données météorologiques sont prometteuses, tant celle de la modélisation statistique que celle de type mécaniste. On a constaté une certaine hétérogénéité spatiale chez les populations de moustiques selon certains éléments probants, ce qui confirme qu'elles peuvent s'adapter aux conditions météorologiques locales. La modulation de la température ou des précipitations attribuable à la topographie locale qui modifie sensiblement I'habitat des moustiques immatures $(16,26)$ en est probablement un facteur explicatif. Ainsi, le fait de prendre en compte la variation topographique locale dans les modèles statistiques améliore leur rendement (22-24).

Une autre approche de la validation, davantage axée sur la santé publique, permet d'estimer la probabilité selon laquelle le modèle prévoit correctement des conditions favorables à une éclosion par opposition à des conditions non favorables (également appelée " aptitudes prévisionnelles " pour ce qui est du modèle de prévisions fondées sur les données météorologiques). L'objectif que vise l'Organisation mondiale de la Santé pour les prédictions concernant la malaria est de pouvoir, de façon acceptable, prédire $60 \%$ des éclosions de malaria dans les deux semaines (13). Un certain nombre de paramètres servent à définir cette capacité, y compris les paramètres basés sur les caractéristiques de l'opérateur à la réception (27), qui quantifient la capacité du modèle à faire preuve d'une sensibilité acceptable (c'est-à-dire un faible nombre de faux négatifs - de sorte que peu d'éclosions n'auront pas été prédites) et d'une spécificité également acceptable (c'est-à-dire un faible nombre de faux positifs - de sorte que peu de fausses alarmes auront été lancées). Le niveau de tolérance au fait de ne pas prédire certaines éclosions ou, a contrario, de lancer de fausses alarmes est une décision que les professionnels de la santé publique et les décideurs politiques doivent assumer, car c'est à eux qu'incombe le fait d'établir le modèle de services de santé publique souhaité.

\section{Discussion}

Le Groupe d'experts intergouvernemental sur l'évolution du climat a fait valoir la nécessité de disposer de systèmes d'alertes précoces comme les prédictions du risque basées sur les prévisions métérologiques pour détecter les éclosions de maladie transmise par les moustiques. Plusieurs modèles de 
prédictions du risque basées sur les prévisions métérologiques ont été mis au point au Canada pour le virus du Nil occidental, dont la fréquence des éclosions est susceptible d'augmenter en raison des changements climatiques anticipés. La recherche initiale sur la validation laisse entendre que ces modèles sont très prometteurs, car ils ont le potentiel de fournir des prévisions à court terme sur le risque (c'est-à-dire avec une ou quelques semaines d'avance). Bien que ce type de prévisions puisse être associé à des délais trop courts pour permettre de déclencher des mesures proactives de réduction ayant pour but d'éradiquer les moustiques immatures (agents larvicides), elles permettraient en revanche l'implémentation de deux mesures : contrôle et réduction des populations de moustiques adultes (agents adulticides) et aux alertes lancées auprès du public afin de renforcer l'adoption de méthodes de protection personnelle. En général, le taux d'adoption de méthodes de protection personnelle est faible (28-30), en partie parce que le public considère que le risque est faible. Les prévisions fondées sur les données météorologiques offrent la possibilité de sensibiliser le grand public et de mieux lui faire comprendre le risque, ce qui susciterait l'adoption de mesures de protection personnelles à des endroits et à des époques où le risque imminent est élevé.

Les prévisions fondées sur les données météorologiques en ce qui concerne le virus du Nil occidental seraient un outil utile à une intervention en matière de santé publique qui permettrait de mieux protéger les Canadiens contre les maladies, émergentes et réémergentes, transmises par les moustiques en les avertissant au plus tôt de l'arrivée d'éclosions imminentes. Il y a cependant des limites à leur application. Premièrement, ce type de prévision ne peut remplacer la surveillance des cas humains, des moustiques et des animaux sentinelles, car des facteurs qui ne dépendent aucunement de la température (ou qui ne lui sont qu'indirectement associés) peuvent être à l'origine d'éclosions. Ces facteurs peuvent comprendre des variations de l'immunité du troupeau au sein d'un plus vaste ensemble de populations d'hôtes sauvages ou la présence de souches émergentes de pathogènes associés aux maladies transmises par les moustiques (4). Il est également prudent de maintenir la surveillance des moustiques afin de valider régulièrement les modèles prévisionnels. Deuxièmement, les signes hâtifs en cas de risque imminent lié au maladies transmises par les moustiques, fournis par les modèles prévisionnels, exigent que les systèmes, les méthodes et les mesures de santé publique puissent réagir rapidement à ces risques, ce qui est le cas dans la plupart des territoires de compétence pratiquant ce type d'intervention en santé publique.

Prédire les éclosions de virus du Nil occidental et d'autres maladies transmises par les moustiques à l'échelle nationale est une possibilité que notre pays doit envisager à l'avenir. La recherche requise avant cette mise en œuvre comprend une validation et une évaluation prospectives des aptitudes prévisionnelles des modèles (notamment une explication de la façon dont le résultat peut varier d'une région à l'autre), leur adoption et leur application par les utilisateurs finaux et l'élaboration de méthodes permettant de mieux communiquer ce type de risque au public. L'atteinte de ces objectifs exigera des études bien documentées dans un champ concerté et en laboratoire qui seront dirigées par des organismes locaux, provinciaux ou territoriaux et fédéraux ainsi que des simulations informatiques qui seront menées par ces mêmes organismes qui œuvrent en collaboration avec des entomologistes, des écologistes, des épidémiologistes et des mathématiciens rattachés à des institutions de recherche universitaire.

À ce jour, les prédictions du risque basées sur les prévisions métérologiques ont seulement servi aux études sur le virus du Nil occidental au Canada parce qu'il n'existe que très peu de connaissances sur l'écologie d'autres maladies transmises par les moustiques qui sont endémiques au Canada (par exemple, les virus du sérogroupe Californie) et très peu de données sur la surveillance systématique avec lesquelles calibrer et valider les modèles. Mais cet état de fait change et ce sera à l'avenir chose possible grâce à davantage de données qui auront été recueillies de manière systématique.

En général, les modèles fondés sur les données météorologiques sont associés à de meilleures aptitudes prévisionnelles sur de courtes échelles de temps (c'est-à-dire des prédictions à court terme ou prolongées de quelques jours à une semaine) (21) et à de moins bonnes aptitudes prévisionnelles sur une plus longue durée (c'est-à-dire des prédictions à long terme ou saisonnières dont la durée varie de quelques semaines à quelques mois) (31). Cependant, certains modèles prévisionnels à grande portée ont atteint la cible fixée par l'Organisation mondiale de la Santé de $60 \%$ d'aptitudes en matière de prévisions (32). Les modèles de prédictions du risque basées sur les prévisions métérologiques' qui ont le mieux réussi à prévoir les variations de l'amplitude du risque chaque saison, comme la malaria, sont ceux dont l'amplitude dépend du phénomène climatique mondial cyclique, surtout le phénomène d'oscillation australe El Niño (33). Ce genre de modèle prévisionnel n'a pas encore fait l'objet d'une exploration pour le virus du Nil occidental au Canada, notamment à cause des courtes (près de 10 ans) séries chronologiques des données de surveillance (34).

\section{Conclusion}

La mise en œuvre future des prévisions fondées sur les données météorologiques du virus du Nil occidental et d'autres maladies transmises par les moustiques permettra de raccourcir le délai avant le lancement d'alertes pour des éclosions imminentes. Comme on s'attend à ce que la fréquence des éclosions de maladies transmises par les moustiques augmente avec les changements climatiques, ces alertes permettront une intervention rapide et précise en santé publique doublée d'une réduction concomitante des répercussions sur la santé publique. L'adoption de modèles de prédictions du risque basées sur les prévisions métérologiques et de leur mise en œuvre par les instances de santé publique au Canada dépend de plus 
amples validations et évaluations des aptitudes prévisionnelles de ces modèles, ainsi que de l'exploration du degré avec lequel les modèles prévisionnels doivent être calibrés selon les régions. L'élaboration de prévisions fondées sur les données météorologiques pour d'autres maladies endémiques et émergentes transmises par les moustiques devrait être réalisée grâce à de meilleures connaissances sur l'écologie de ces maladies et à davantage de surveillance systématique.

\section{Déclaration des auteurs}

$\mathrm{NHO}$ est le principal auteur de l'article, $\mathrm{NHO}$ et $\mathrm{AL}$ ont conceptualisé l'article, tous les auteurs (NHO, LRL, AL, APM, HZ, $\mathrm{HZ}$ ) ont contribué à sa rédaction.

\section{Conflit d'intérêts}

Aucun.

\section{Financement}

Le présent travail a été soutenu par l'Agence de la santé publique du Canada.

\section{Références}

1. Smith KR, Woodward A, Campbell-Lendrum D, Chadee DD, Honda Y, Liu Q, Olwoch JM, Revich B, Sauerborn R, Aranda C, Berry H, Butler C, Chafe Z, Cushing L, Ebi KL, Kjellstrom T, Kovats S, Lindsay G, Lipp E, McMichael T, Murray V, Sankoh O, O'Neill M, Shonkoff SB, Sutherland J, Yamamoto S. Climate Change 2014: Impacts, Adaptation, and Vulnerability. Part A: Global and Sectoral Aspects. Contribution of Working Group II to the Fifth Assessment Report of the Intergovernmental Panel on Climate Change. Cambridge, UK and New York, USA: Cambridge University Press; 2014. Human Health: Impacts, Adaptation, and Co-Benefits. p. 709-54. www.ipcc.ch/site/assets/ uploads/2018/02/WGIIAR5-Chap11_FINAL.pdf

2. Zinszer K, Verma AD, Charland K, Brewer TF, Brownstein JS, Sun Z, Buckeridge DL. A scoping review of malaria forecasting: past work and future directions. BMJ Open 2012 Nov;2(6):e001992. DOl PubMed

3. Semenza JC. Prototype early warning systems for vector-borne diseases in Europe. Int J Environ Res Public Health 2015 Jun;12(6):6333-51. DOl PubMed

4. Agence de la santé publique du Canada. Surveillance du virus du Nil occidental. Ottawa (ON): ASPC; 2018. (Accédé 2018-10-25). https://www.canada.ca/fr/sante-publique/ services/maladies/virus-nil-occidental/surveillance-virus-niloccidental.html

5. Kulkarni MA, Lecocq AC, Artsob H, Drebot MA, Ogden NH. Epidemiology and aetiology of encephalitis in Canada, 19942008: a case for undiagnosed arboviral agents? Epidemiol Infect 2013 Nov;141(11):2243-55. DOI
6. Drebot MA. Bunyavirus transmis par les moustiques émergents au Canada. Relevé des maladies transmissibles au Canada. 2015 Jun;41(6):133-41. DOI PubMed

7. Ludwig A, Zheng H, Vrbova L, Drebot MA, Iranpour $M$, Lindsay LR. Augmentation du risque de maladies endémiques transmises par des moustiques au Canada en raison du changement climatique. Relevé des maladies transmissibles au Canada 2019;45(4):99-107. DOI

8. Ng V, Rees EE, Lindsay LR, Drebot MA, Brownstone T, Sadeghieh T, Khan SU. Les changements climatiques pourraient-ils entraîner la propagation de maladies exotiques transmises par les moustiques au Canada? Relevé des maladies transmissibles au Canada 2019;45(4):108-18. DOI

9. Ogden NH, Gachon P. Changements climatiques et maladies infectieuses: À quoi pouvons-nous nous attendre? Relevé des maladies transmissibles au Canada 2019;45(4):83-8.DOI

10. Githeko AK, Lindsay SW, Confalonieri UE, Patz JA. Climate change and vector-borne diseases: a regional analysis. Bull World Health Organ 2000;78(9):1136-47. PubMed

11. Reisen W, Brault AC. West Nile virus in North America: perspectives on epidemiology and intervention. Pest Manag Sci 2007 Jul;63(7):641-6. DOl PubMed

12. Agence de la santé publique du Canada. Prévention du virus du Nil occidental. Ottawa (ON) : ASPC; 2018. https://www. canada.ca/fr/sante-publique/services/maladies/virus-niloccidental/prevention-virus-nil-occidental.html

13. World Health Organization. 2005-2010 Roll Back Malaria: Global strategic plan. Geneva (CH): WHO; 2005. www1. paho.org/hq/dmdocuments/2010/mal-2005-cor-1.pdf

14. Ogden NH, Lindsay LR. Effects of climate and climate change on vectors and vector-borne diseases: ticks are different. Trends Parasitol 2016 Aug;32(8):646-56. DOI PubMed

15. Government of Saskatchewan. West Nile Virus Surveillance Report, 2018: June 23. http://publications.gov.sk.ca/ documents/13/107167-West-Nile-Virus-Surveillance-ReportWeek-of-June-23-2018.pdf

16. Public Health Ontario. West Nile Virus surveillance. www publichealthontario.ca/en/dataandanalytics/pages/wnv.aspx

17. Institut national de santé publique du Québec. Rapport de surveillance du virus du Nil occidental et autres arbovirus transmis par les moustiques au Québec. www.inspq.qc.ca/ sites/default/files/publications/2455_surveillance_virus_nil_ occidental_arborovius.pdf

18. Hoshen MB, Morse AP. A weather-driven model of malaria transmission. Malar J 2004 Sep;3:32. DOl PubMed

19. Diouf I, Rodriguez-Fonseca B, Deme A, Caminade C, Morse AP, Cisse M, Sy I, Dia I, Ermert V, Ndione JA, Gaye AT. Comparison of malaria simulations driven by meteorological observations and reanalysis products in Senegal. Int J Environ Res Public Health 2017 Sep;14(10):E1119. DOI PubMed

20. Yu D, Madras N, Zhu H. Temperature-driven population abundance model for Culex pipiens and Culex restuans (Diptera: culicidae). J Theor Biol 2018 Apr;443:28-38. DOI PubMed 
21. Wang J, Ogden NH, Zhu H. The impact of weather conditions on Culex pipiens and Culex restuans (Diptera: Culicidae) abundance: a case study in Peel Region. J Med Entomol. 2011;48(2):468-75. PubMed

22. Ripoche M, Campagna C, Ludwig A, Ogden NH, Leighton PA. Short-term forecasting of daily abundance of West Nile virus vectors Culex pipiens-restuans and Aedes vexans based on weather conditions and larvicide use in southern Québec (Canada). J Med Entomol 2019;56(3):1-14. DOI

23. Chen CC, Epp T, Jenkins E, Waldner C, Curry PS, Soos C. Modeling monthly variation of Culex tarsalis (Diptera: Culicidae) abundance and West Nile Virus infection rate in the Canadian Prairies. Int J Environ Res Public Health 2013 Jul;10(7):3033-51. DOl PubMed

24. Gao X, Cao YR, Ogden N, Aubin L, Zhu HP. Mixture Markov regression model with application to mosquito surveillance data analysis. Biom J 2017 May;59(3):462-77. DOI PubMed

25. Laboratory of Mathematical Parallel Systems. Mosquito Abundance and West Nile Risk Yearly Forecasting for Peel Region. www.lamps.yorku.ca/yearly-forecast-graphs

26. Yusa A, Berry P, J Cheng J, Ogden N, Bonsal B, Stewart R, Waldick R. Climate change, drought and human health in Canada. Int J Environ Res Public Health 2015 Jul;12(7):8359_ 412. DOl PubMed

27. MacLeod DA, Jones A, Di Giuseppe F, Caminade C, Morse AP. Demonstration of successful malaria forecasts for Botswana using an operational seasonal climate model. Environ Res Lett. 2015;10(4): 044005. DOI
28. Centers for Disease Control and Prevention (CDC).

Knowledge, attitudes, and behaviors about West Nile virus-Connecticut, 2002. MMWR Morb Mortal Wkly Rep 2003 Sep;52(37):886-8. www.cdc.gov/mmwr/preview/mmwrhtml/ mm5237a4.htm PubMed

29. Wilson SD, Varia M, Lior LY; Field Epidemiology Summer Course. West Nile Virus: the buzz on Ottawa residents' awareness, attitudes and practices. Can J Public Health 2005 Mar-Apr;96(2):109-13. PubMed

30. Trumbo CW, Harper R. Perceptual influences on self-protective behavior for West Nile virus, a survey in Colorado, USA. BMC Public Health 2015 Jun;15:557. DOI PubMed

31. Gouvernement du Canada. Habileté du système de prévisions déterministes. 2019. https://meteo.gc.ca/saisons/ skill_f.html

32. Lauderdale JM, Caminade $C$, Heath AE, Jones AE, MacLeod DA, Gouda KC, Murty US, Goswami P, Mutheneni SR, Morse AP. Towards seasonal forecasting of malaria in India. Malar J 2014 Aug;13:310. DOI PubMed

33. Thomson MC, Doblas-Reyes FJ, Mason SJ, Hagedorn R, Connor SJ, Phindela T, Morse AP, Palmer TN. Malaria early warnings based on seasonal climate forecasts from multi-model ensembles. Nature 2006 Feb;439(7076):576-9. DOI PubMed

34. Manore CA, Davis J, Christofferson RC, Wesson D, Hyman JM, Mores CN. Towards an early warning system for forecasting human west nile virus incidence. PLoS Curr 2014 Mar;6. DOl PubMed 